
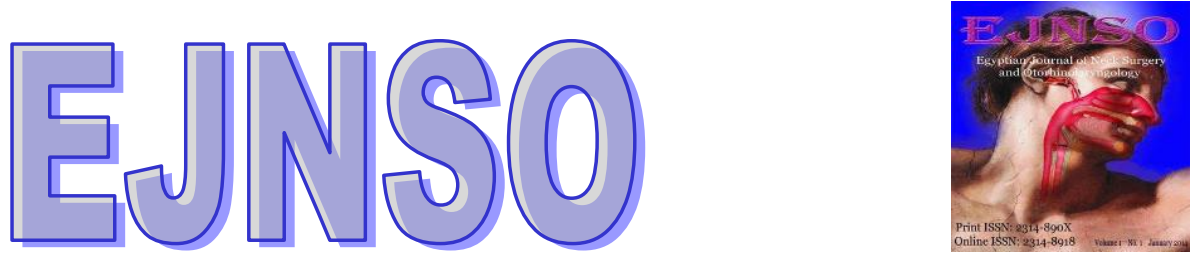

\title{
Squamous Papilloma: An Unusual Presentation as a Large Pedunculated Mass over Buccal Mucosa
}

\author{
R. K. Mundra. Richi Sinha. Richa Agrawal \\ Department of Otorhinolaryngology Head and Neck Surgery, MGM Medical College \& MY Hospitals, Indore, Madhya Pradesh, \\ India.
}

Received: August $13^{\text {th }}, 2013$

Revised: August $29^{\text {th }}, 2013$

Accepted: September $20^{\text {th }}, 2013$

Keywords: papilloma, benign neoplasm, oral cavity, human papilloma virus

\begin{abstract}
Oral squamous papilloma, a benign proliferation of stratified squamous epithelium commonly involves palate. It is cauliflower-like projection, seldom larger than $1.0 \mathrm{~cm}$. We report a rare case of large $2.8 \times 2.7 \times 2.0 \mathrm{~cm}$ smooth pedunculated squamous papilloma involving buccal mucosa prolapsing outside oral cavity. The patient underwent surgical excision with complete resolution of symptoms. No such case report was found on searching the available literature.
\end{abstract}

\section{INTRODUCTION}

Various unique squamous-derived entities of the oral cavity and oropharynx exist; however, one of the most frequently occurring conditions is squamous papilloma ${ }^{1}$. The squamous papillomas are benign exophytic masses. It is an innocuous lesion that is neither transmissible nor threatening ${ }^{2}$. They appear as pedunculated or sessile, pink-to-white colored cauliflower- like projections arising from the mucosal surface of commonly the palate, uvula, tongue and lips. Most of the papillomas measure less than $1.0 \mathrm{~cm}$. Many are only 3 to 4 $\mathrm{mm}^{3}$. They rarely cause symptoms. Many considered its pathogenesis as being from the human papilloma virus (HPV). But recent literature suggests that presence of HPV may be merely an incidental finding unrelated to the development of a squamous papilloma ${ }^{4}$. We report an interesting case of a large pedunculated oral squamous papilloma which is prolapsable.

\section{CASE REPORT}

A 70-year-old male reported to the Department of Otorhinolaryngology with the chief complaint of mass on the buccal mucosa for one year causing difficulty in speech and eating. History regarding the growth revealed that it was first seen one year back following trauma by cheek bite as a slow-growing nontender papule. It was initially sessile but became pedunculated after 6-7 months, when patient was able to protrude it outside the oral cavity (Fig.1) to help him speak and eat. The present lesion was single, $2.8 \times 2.7 \times 2.0 \mathrm{~cm}$ globular mass, grey-white to grey-brown in color with smooth surface, soft to firm in consistency, and arising from the buccal mucosa of left side (Fig.2). Patient also had history of tobacco chewing for 30 years.

Based on the history, clinical features and nature of the growth a provisional diagnosis of benign tumor was considered. Differential diagnosis included fibroma, lipoma and papilloma.

Patient was subjected to complete hematological examination and all the parameters were within normal limits. The lesion was totally excised (Fig.3) from the buccal mucosa under local anesthesia with electrocautery, leading to complete resolution of symptoms. Histopathology revealed characteristic findings of a squamous papilloma, including hyperplastic squamous epithelial lining with acanthosis, elongation of rete ridges and papillary projections (Fig.4). There was no evidence of malignancy. Patient's postoperative course was uneventful. 

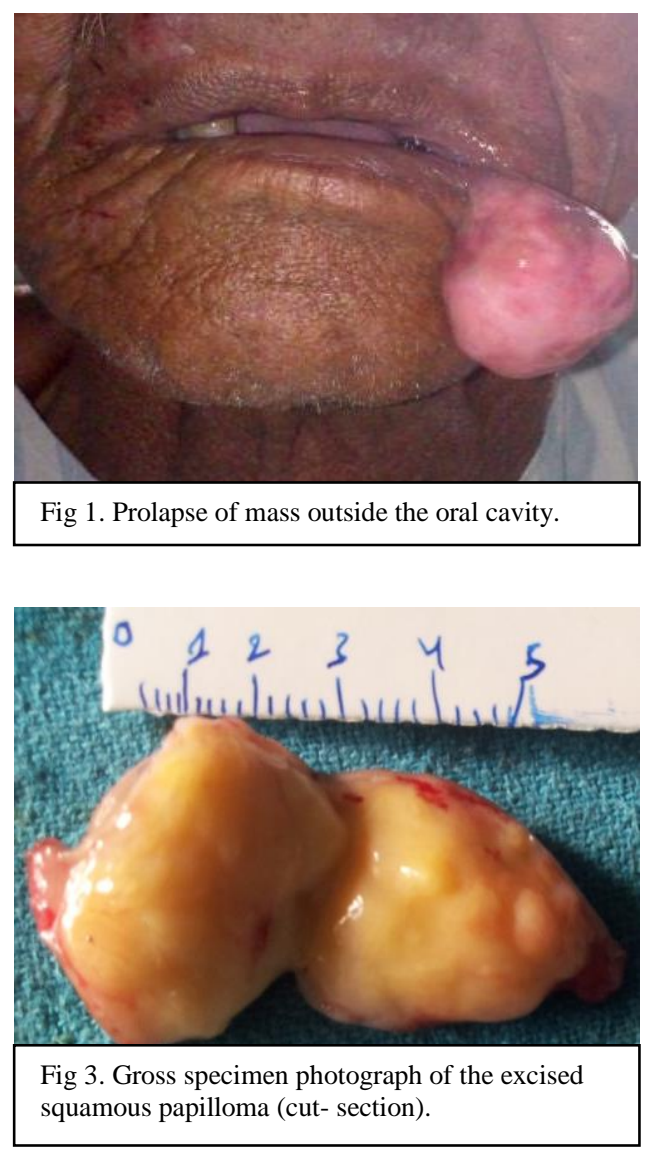

\section{DISCUSSION}

Oral squamous papillomas are benign mucosal neoplasms. They result from viral infection of the epithelium, especially from HPV. HPV encompasses a group of doublestranded DNA viruses of the papova virus subgroup A. The most commonly involved subtypes are HPV-6 and HPV-11, which are not associated with malignancy or pre-cancer ${ }^{3}$.

Oral and oropharyngeal squamous papilloma occur mainly between 30 and 50 years of age and may occur below 10 years 5 . In our case it presented at 70 years of age. Although they have a predilection for palate and uvula accounting for approximately one third of all lesions ${ }^{6}$, here it was arising from the buccal mucosa. These lesions appear as pink-to-white exophytic granular or cauliflower-like surface alterations usually measuring less than $1 \mathrm{~cm}$ and are asymptomatic ${ }^{4}$. However this patient had difficulty in speaking and eating. Another interesting fact was that, the patient could expel the mass in and out of oral cavity, which temporarily relieved his symptoms.

Squamous papillomas are traditionally divided into two types: isolated-
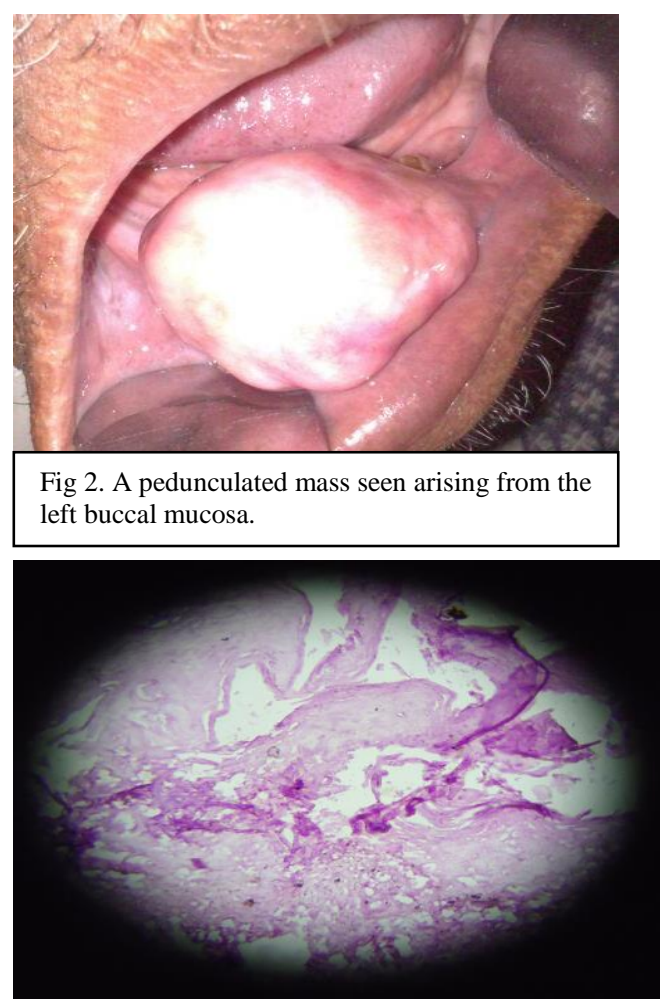

Fig 4. Microscopic photograph in high power with hematoxylin and eosin stained sections of oral squamous papilloma, showing the presence of papillary finger like projections. solitary and multiple-recurring. The former is usually found in an adult's oral cavity, while the latter is found in a child's laryngotracheobronchial complex. The patients who are human immunodeficiency virus positive often have multiple oral lesions. Malignant transformation of a papilloma is more common in the multiple-recurring type ${ }^{4}$.

Squamous papilloma typically has a narrow stalk below a mass with numerous surface projections, often characterized as finger-like. Submucosal fibrovascular connective tissues are contiguous with the stroma of the stalk, the body of the mass and the surface projections. Scattered chronic inflammatory cells in small numbers are common in the stroma, presumably from chronic low-grade trauma to the lesion.

The surface keratin is often quite thickened, usually with parakeratin. The covering squamous epithelium shows a normal maturation pattern, although occasional papillomas demonstrate pronounced basilar hyperplasia and mild mitotic activity whith could be mistaken for mild epithelial 
dysplasia. Koilocytes (HPV-altered epithelial cells with perinuclear clear spaces and nuclear pyknosis) may or may not be found in the superficial layers of the epithelium ${ }^{7}$.

The oral squamous papilloma differs from papillomas elsewhere in a few important ways. While most of these growths are noncancerous in nature, a growth within the mouth does have a slightly higher likelihood of developing malignancy. Oral papillomas also occur in greater frequency, posing a potential respiratory risk if they overpopulate the throat.

Papillomas found on the nasal or throat regions although sharing the same clinical features and histology as oral papillomas, differ in that usually more than one lesion is present, they proliferate continuously over time and often recur. The rare intraoral verruca vulgaris, condyloma acuminatum, and focal epithelial hyperplasia resemble papillomas and microscopic examination may be required to distinguish between them. Large papillomas may resemble early verrucous carcinoma ${ }^{3}$.

Conservative surgical excision including the base of the lesion by either routine excision or laser ablation is adequate treatment for squamous papilloma, and recurrence is unlikely, except in patients infected with human immunodeficiency virus ${ }^{4}$.

Other treatment modalities include electrocautery, cryosurgery, and intralesional injections of interferon. Frequently lesions left untreated for years, reported no malignant transformation or dissemination to other parts of the oral cavity ${ }^{7}$.

\section{CONFLICT OF INTEREST: None REFERENCES}

1. Flint PW, Haughey BH, Lund VJ, et al. Benign neoplasms, Cummings Otolaryngology-Head and Neck Surgery, Elsevier Mosby, Philadelphia, Pa, USA, 5th edition, 2010.

2. Squamous Papilloma (Benign Epithelial Tumors of Mucosa) by Neuron arc, November 23,2012.(http://www.neuronarc.com/squamous -papilloma-benign-epithelial-tumors-of mucosa.html\#Hh1CfJalZPKqgdJQ.99)

3. Kumar AN, Rajeswaran R, Kumar HH. Large Squamous Papilloma of Buccal Mucosa. Journal of case reports 2012;2(2):110-113.

4. Jaju PP, Suvarna PV, Desai RS. Squamous Papilloma: Case Report and Review of Literature. International Journal of Oral Sciences, 2(4): 222-225, 2010.

5. Kumar BP, Khaitan T, Ramaswamy $P$. Squamous Papilloma. International Journal of Stomatology \& Occlusion Medicine, March 2013. (Database on Internet)

6. Abbey LM, Page DG, Sawyer DR. The clinical and histopathologic features of a series of 464 oral squamous cell papillomas. Oral Surgery Oral Medicine and Oral Pathology, vol. 49, no. 5, pp. 419-428, 1980.

7. Thomas E. Bond, "Squamous Papilloma", Bond's Book of Oral Diseases, 4th Edition, Maxillofacial Center for Diagnostics \& Research. (database on internet) 